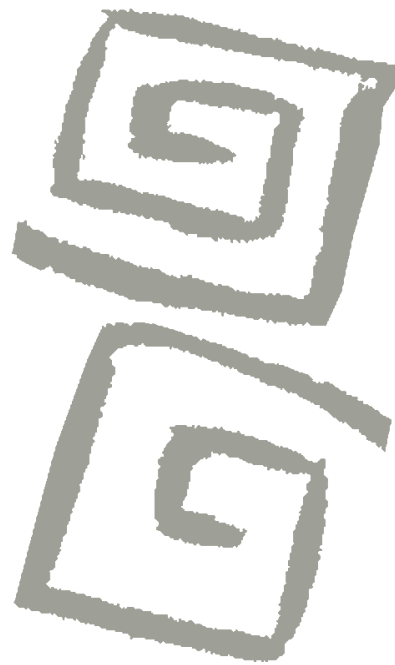

\title{
De la sociología en la medicina a la sociología de la salud colectiva: apuntes para un necesario ejercicio de reflexividad
}

\author{
From sociology in medicine to the sociology of \\ collective health: contributions toward a necessary \\ reflexivity
}

Roberto Castro ${ }^{1}$

${ }^{1}$ Doctor en Sociología Médica. Investigador Titular, Centro Regional de Investigacione Multidisciplinarias (CRIM), Universidad Nacional Autónoma de México (UNAM), Cuernavaca, México. rcastro@correo.crim.unam.mx
RESUMEN En este texto se aborda la distinción entre la sociología en la medicina (colaboradora de las instituciones de salud) y la sociología de la medicina (independiente de las instituciones de salud). Se argumenta que, si es consecuente, la sociología en la medicina deviene sociología de la medicina. Como ejemplo, se discute el caso de los determinantes sociales de la salud que, inicialmente, asumen como no problemática la realidad ontológica de la salud-enfermedad y luego problematizan el concepto de salud-enfermedad y muestran que estudiar los determinantes exige estudiar también los determinantes de los procesos de construcción social de la enfermedad. Se muestra la ineludible necesidad de objetivar la propia salud colectiva, es decir, de aplicar las herramientas de la sociología de manera que podamos poner bajo examen los llamados factores objetivos de la determinación de la salud-enfermedad, el carácter socialmente construido de las categorías de conocimiento, y las luchas y relaciones de poder que determinan la viabilidad o no de tales categorías.

PALABRAS CLAVES Sociología Médica; Teoría Social; Medicalización; Desigualdades en la Salud.

\begin{abstract}
This text looks at the difference between sociology in medicine (collaborator of health institutions) and the sociology of medicine (independent of health institutions). If consistent, sociology in medicine should become a sociology of medicine. As an example, it is shown how the study of the social determinants of health and illness begins by assuming non-problematically the ontological reality of health and illness, but ends up problematizing the very concept of health-disease, demonstrating that the study of health determinants also requires the study of the determinants of the social construction of disease. The urgent necessity of objectifying collective health itself is argued. By applying sociological tools we can examine the so-called objective factors in the determination of health and disease, the socially constructed nature of these categories of knowledge, and the struggles and power relations that determine whether or not such categories are viable.
\end{abstract}

KEY WORDS Medical Sociology; Social Theory; Medicalization; Health Inequalities. 


\section{INTRODUCCIÓN}

En un trabajo de hace casi 60 años, que se volvió un clásico ${ }^{(1)}$, Straus propuso diferenciar entre sociología en y sociología de la medicina. La primera quedó definida como la investigación "colaborativa" que se hace desde y con la medicina, y que tiene como finalidad desarrollar la agenda de la salud. La segunda, en cambio, se refiere a aquella que es realizada "desde posiciones independientes fuera del contexto médico formal” ${ }^{\text {(1 p.203) }}$, y tiene como objetivo hacer de la medicina, sus instituciones, saberes y prácticas, su objeto de estudio.

En este trabajo mostraremos que dicha diferencia es útil solo hasta cierto punto, ya que la llamada sociología en la medicina, si es fiel a sí misma, propende a devenir en sociología de la medicina, por las exigencias que impone la reflexividad a la que está obligada la disciplina. Un buen ejemplo para ilustrar este argumento es el caso del estudio de los determinantes sociales de la salud-enfermedad: en la construcción de este objeto de estudio las tareas investigativas empiezan bajo la forma de sociología en la medicina, esto es, como una colaboración con la salud pública/colectiva, mirando las cosas desde la perspectiva del experto que hace de la salud-enfermedad su objeto de interés. Sin embargo, una vez puesta en marcha la indagatoria sociológica, es inevitable evolucionar hacia una sociología de la medicina, es decir, que focaliza la indagación en el carácter performativo de las actuaciones profesionales sobre lo que se denomina enfermedad y, sobre todo, en las luchas que subyacen al interior del campo médico o de la salud. Veremos que una sociología de la medicina apunta a una tarea ineludible: la necesidad de ir más allá de las proclamas que reivindican la centralidad de las ciencias sociales en la salud colectiva para dar paso específicamente a una sociología de la salud colectiva. A los efectos de este trabajo, adoptamos la caracterización de salud colectiva propuesta por Nunes ${ }^{(2)}$, en tanto corriente de pensamiento, práctica teórica y movimiento social, producto de la evolución del pensamiento social latinoamericano en salud y resultado de la incorporación de sus vertientes más críticas.

Quizá cabe decir, en un plano epistemológico, que la disputa entre el positivismo y las ciencias sociales ha quedado saldada (no así en otros planos, como el de la administración académica o el de la financiación pública de la investigación científica). Con el auge de los enfoques interpretativos desde la década de 1980 (que reivindican la construcción del objeto de las ciencias sociales a partir del sentido de la acción y sus significados), difícilmente se puede argumentar que el método científico de las ciencias sociales debe tratar de asemejarse al de las ciencias físicas, naturales y exactas ${ }^{(3)}$. En todo caso, tales requerimientos se le formulan a las ciencias sociales desde otros campos del conocimiento, como la biomedicina o la salud pública, o desde las burocracias académicas, pero se trata de exigencias externas a la disciplina que hablan de las luchas que se libran al interior del campo científico.

Pero aun tratándose de disciplinas en las que la relación sujeto-objeto es de naturaleza enteramente diferente a la que puede existir en las ciencias naturales, las ciencias sociales siguen siendo ciencias ${ }^{(4)}$. Por tanto, no puede minimizarse la importancia de desarrollar y utilizar marcos teóricos y métodos de análisis especializados, que deben implementarse, en la construcción de sus objetos de estudio, con rigor y mediante procedimientos que deben quedar sujetos al escrutinio y la valoración de los pares. El postulado de Popper ${ }^{(5)}$ de que el atributo fundamental del método científico es su carácter público aplica sin reserva para las ciencias sociales. Para que no quede reducida a una mera sociología espontánea, indiferenciable por tanto del sentido común, el método de las ciencias sociales aplicadas a la salud debe tener carácter público, esto es, debe permitir que sus conceptos, pasos, técnicas y evidencias puedan someterse a la auditoría de los pares, a diferencia de las opiniones de sobremesa, que no están sujetas a ningún tipo de verificación controlada ${ }^{(6)}$. $\mathrm{Y}$, al mismo tiempo, debe permitir a los 
especialistas aplicar sobre sí mismos las herramientas de investigación que aplican sobre los diversos objetos que construyen. Ello, no solo porque dicho ejercicio es indispensable como estrategia de ruptura, sino porque la superación de los obstáculos que impiden el pleno desarrollo de la salud colectiva resulta imposible si no se objetiva científicamente a esta última. Por tanto, no es irrelevante la exigencia -impulsada sobre todo desde las universidades- de reglamentar el acceso de los aspirantes a ser certificados como expertos en ciencias sociales, básicamente mediante la obligación que se les impone de acreditar que conocen bien las reglas básicas del juego: las reglas del método científico de las ciencias sociales. Y no es inconsecuente la flexibilización de este criterio.

La confluencia entre ciencias sociales y salud en América Latina ha conocido diversas denominaciones, que reflejan las luchas y los posicionamientos ideológicos y políticos específicos en esta materia. Así, en diferentes momentos, sobre todo desde el ámbito académico, se ha hablado de ciencias de la conducta y medicina, sociología y antropología médicas, medicina social y salud colectiva. Incluso, bajo el impulso de la Organización Mundial de la Salud (OMS), se llegó a hablar de la "nueva salud pública", que veía en lo "poblacional" una coincidencia de niveles de análisis con las ciencias sociales y buscaba diferenciarse de la salud pública más tradicional ${ }^{(7)}$.

No es difícil advertir una gran variabilidad en la calidad científica y en el alcance crítico (en realidad, ambas cosas van de la mano) de lo que se publica en el área de ciencias sociales y salud en América Latina. Tal disparidad no es únicamente el resultado de una falta de rigor de algunos trabajos, sino que obedece también a una situación estructural, discernible sociológicamente ${ }^{(8),(9)}$.

Por una parte, en efecto, se ha desarrollado una suerte de sociología médica relativamente autónoma (y, desde luego, sujeta a sus propias luchas internas) pero que, albergada en algunas universidades y centros de investigación relativamente autónomos, suele permanecer ajena al sector salud, desde donde también tiende a ser ignorada, tanto por desconocimiento como por omisión ${ }^{(10)}$. Se trata entonces de un quehacer que en ocasiones puede alcanzar un alto nivel de desarrollo teórico, metodológico y crítico, pero que tiene poco impacto en el ámbito de las políticas y los programas de salud.

Por otra parte, desde las instituciones oficiales de salud pública se incentiva el desarrollo de una ciencia social "domesticada", subalterna, con fines meramente instrumentales, sin mayor potencial crítico. Es un quehacer con más impacto en el sector, pero que opera siempre dentro de los estrechos límites que determina el propio establishment médico.

Esta dicotomía, que cuenta con bases materiales que la reproducen -cada enfoque se ancla en instituciones públicas o universitarias específicas, o en comunidades epistémicas particulares-, se halla en el origen de la dificultad que se observa hasta ahora para aplicar el principio de la reflexividad ${ }^{(11)}$ dentro del mundo de los estudios de sociología médica o de la sociología de la salud. Ahí donde la agenda de las ciencias sociales en salud la dicta el establishment médico, la construcción del objeto difícilmente puede incluir críticamente al propio campo médico $^{[a]}$; en esos casos, suele constatarse que el aporte de las ciencias sociales no va más allá de ciertas descripciones de contexto o de la implementación de cierto tipo de estudios cualitativos no siempre diferenciables de la sociología espontánea.

Como veremos ahora, un buen ejemplo para mostrar la continuidad que existe entre una sociología en la salud y una sociología de la salud es el estudio de los determinantes sociales de la salud-enfermedad, que puede comenzar como sociología en, es decir, al servicio de la agenda de las políticas de salud pública, pero que omitirá muy importantes aspectos en la construcción del objeto, a menos que acierte en objetivar a los propios sujetos objetivantes. La salud colectiva está en condiciones de llevar a cabo su propia objetivación y, con ello, acceder a un mejor conocimiento (y eventual transformación) de las condiciones de posibilidad que estructuran el tipo de conocimiento que produce. 


\section{DE CÓMO SE TRANSITA EMPÍRICAMENTE DE UNA SOCIOLOGÍA EN SALUD A UNA SOCIOLOGÍA DE LA SALUD}

En otro trabajo hemos mostrado ya cómo el estudio social de los determinantes de la salud-enfermedad admite tres aproximaciones $^{(14)}$. La primera, referente a una sociología en la medicina, consiste en la diferenciación de los determinantes sociales por niveles, desde los de más amplio alcance (como la globalización y el cambio climático), pasando por los estructurales (como el modo de producción, la desigualdad de clase y las determinaciones de género), los de nivel intermedio (el proceso de trabajo y el apoyo social) y, finalmente, a nivel individual, los llamados "estilos de vida". Sobre esta materia, al tratar de identificar cuáles son las principales disputas en torno al objeto, hay que señalar la controversia existente entre la propuesta analítica de la $\mathrm{OMS}^{(15)}$ y la formulada por la Asociación Latinoamericana de Medicina Social (ALAMES) ${ }^{(16)}$ y $\operatorname{otros}^{(17),(18)}$. Estas últimas, producidas sobre todo desde espacios académicos, son mucho más agudas que la primera al identificar el origen social -ergo, político, económico, de justicia social- de los determinantes, mientras que la propuesta de la OMS está claramente sujeta a las determinaciones que impone la negociación posible entre los Estados miembros de ese organismo internacional. Y si bien los trabajos críticos constituyen, sin duda, una excelente aproximación al problema de los determinantes, por cuanto insisten en la obligatoriedad de introducir las dimensiones de poder que explican la existencia de los determinantes reconocidos por la OMS, no podemos dejar de coincidir con el señalamiento de otros autores, en el sentido de que bajo este enfoque hace ya mucho tiempo que los principales determinantes están bien identificados y, por tanto, es poco el conocimiento realmente nuevo en esta materia ${ }^{(19)}$. Pero, sobre todo, no podemos dejar de advertir la presunción positivista que subyace a esta clasificación, por cuanto supone que la salud y la enfermedad son conceptos relativamente no-problemáticos para la medicina ni para las ciencias sociales. Es decir, supone que la enfermedad se define básicamente por los criterios objetivos de la ciencia biomédica y asume que esta última es, efectivamente, la perspectiva más autorizada para delimitar las fronteras entre lo normal y lo patológico. Esta jerarquización de los determinantes por niveles de agregación, que va de lo macro a lo micro y que no se explica si no se le ubica adecuadamente dentro del contexto del capitalismo mundial, siendo ya un aporte de las ciencias sociales (por el orden que introduce y porque obliga a descubrir sus interconexiones más allá de la biología) no puede constituir el punto de llegada en lo que se refiere al estudio social de los determinantes de la salud y la enfermedad. Pues, una vez echada a andar la indagatoria sociológica sobre los "determinantes", es inexcusable estudiar también la manera en que las ciencias biomédicas y la práctica médica occidental construyen sus propios objetos de estudio. No hacerlo así supone una complicidad con el enfoque positivista que postula que las enfermedades, como los demás objetos que interesan a la investigación científica, están "ahí afuera" y solo es cuestión de entrar en contacto con ellos para estudiarlos.

La segunda aproximación a los determinantes de la salud-enfermedad presenta, como contribución fundamental, una problematización del concepto mismo de saludenfermedad, y abre paso así al estudio del carácter socialmente construido de este fenómeno. Ejemplos de ello fueron los trabajos realizados desde la teoría de la etiquetación (20),(21),(22),(23), que mostraron, desde diversas perspectivas, que la enfermedad (y la desviación) está lejos de ser un concepto estable, y que su presunción (ergo, su existencia), obedece fundamentalmente a dinámicas interaccionales claramente determinadas por dinámicas de poder. Desde este enfoque se mostró que aquello que Ilamamos "enfermedad" es más el resultado de intensas luchas y negociaciones entre diversos grupos sociales, que el corolario de un objetivo y aséptico proceso de investigación biomédica ${ }^{(24)}$. La profesión médica, señaló Freidson ${ }^{(25)}$, se encuentra activamente comprometida en la medicalización 
de la realidad, lo que se traduce en una constante expansión del horizonte médico: cada vez son más las conductas, los signos y los síntomas que la medicina reclama como objetos de su competencia. De esto se desprende una consecuencia devastadora para el paradigma biomédico clásico: los determinantes de la enfermedad son también de orden político, pues hay que buscarlos básicamente en la actividad clasificatoria de los profesionales de la medicina, particularmente, de los que tienen más poder ${ }^{(26)}$.

Desde luego no puede obviarse el papel que juegan las grandes compañías farmacéuticas en la invención -también Ilamada disease mongering ${ }^{(27)}$ - de nuevas enfermedades, impulsadas por el ánimo de incrementar sus mercados y sus ganancias. Hay que decir que sorprende el relativo silencio que al respecto han guardado las ciencias sociales en la materia. Y si bien sería poco sofisticado sociológicamente pretender que todo se reduce a una cuestión de mercados y ganancias, e ignorar así todos los otros procesos sociales que se asocian al fenómeno de la construcción social de la enfermedad, es insoslayable incorporar esta cuestión de manera central en estas indagatorias.

Junto al disease mongering también hay que incluir como objeto de estudio lo que podríamos denominar la "nueva medicalización" impulsada por ciertas filosofías new age pseudocientíficas que promueven ( $\mathrm{y}$, sobre todo, venden) visiones supuestamente críticas de la medicina alópata convencional, y difunden nociones esotéricas del cuerpo y la salud en términos de "campos de energía", "magnetismo y salud", "foco tonal", etc., las mismas que, a su vez, se traducen en supuestas nuevas "patologías" que hay que combatir ${ }^{[b]}$. La investigación social sobre determinantes de la saludenfermedad eventualmente tendrá que tornar su mirada hacia estos fenómenos paralelos, sin soslayar las estrategias de legitimación institucional a las que están accediendo ${ }^{[c]}$.

La segunda aproximación, entonces, lejos de argumentar que las enfermedades son meras invenciones, o que las categorías de conocimiento médico carecen de un correlato material, lo que hace es problematizar la aparente estabilidad de las categorías médicas, y mostrar que las propias categorías de conocimiento, mediante las cuales se identifica la enfermedad y sus determinantes, son objetos de lucha, y que esta lucha solo es discernible mediante las herramientas de las ciencias sociales ${ }^{[\mathrm{d}]}$.

Pero es necesario ir más allá. El enfoque del construccionismo social, al tiempo que desestabiliza las categorías médicas de conocimiento y muestra su carácter histórico y socialmente negociado, presupone implícita y erróneamente que el especialista en ciencias sociales cuenta con una posición privilegiada para observar los mecanismos de funcionamiento de la maquinaria social, como si tales mecanismos que tan perspicazmente identifica en los demás no operaran sobre sí mismo. Si es posible estudiar los determinantes de la saludenfermedad, y si es posible estudiar también los determinantes de las categorías mismas de salud y enfermedad, debe aún ser posible estudiar los determinantes sociales del quehacer sociológico en salud que nos permiten, o no, llevar a cabo aquellas dos aproximaciones.

\section{HACIA UNA SOCIOLOGÍA DE LA SALUD COLECTIVA}

La salud colectiva ha avanzado en el esfuerzo por objetivarse a sí misma, pero no ha completado la tarea. Es notable la cantidad de reflexión acumulada -que se manifiesta a través de múltiples libros y artículos académicos- acerca de los orígenes y la naturaleza de la salud colectiva, sobre sus retos actuales y el papel de las ciencias sociales en ella; $y$ sobre las dificultades que enfrenta para dejar su lugar subalterno dentro del campo médico o de la salud y acceder a una posición más favorable respecto a las otras disciplinas ${ }^{(2),(32)}$. Es notable, al mismo tiempo, que tales ejercicios suelen quedarse a mitad de camino en el proceso de objetivación al que es necesario someter a la perspectiva analítica que se pretende reivindicar. Como mostró Bourdieu, "una tarea de objetivación solo está científicamente controlada en proporción a la 
objetivación a que ha sido sometido previamente el sujeto de la objetivación”(11 p.160).

Para iniciar este ejercicio de reflexividad, se puede tomar como un dato el contenido de los artículos que reflexionan sobre la relación entre las ciencias sociales y la salud colectiva, es decir, como una expresión de un grupo de actores del campo de la salud, encargados de la objetivación social de los objetos de la salud, que no se han objetivado a sí mismos y que, al decir lo que señalan en esos artículos, dicen algo sobre aquella relación pero, sobre todo, dicen mucho acerca de su posición en el campo y sus puntos de vista así determinados. Por ejemplo, muchos artículos señalan la relación de subordinación que las ciencias sociales ha mantenido con las ciencias biomédicas, sobre todo, en el espacio (de acción política) de la salud colectiva/salud pública y, en buena medida, en el espacio (académico) de la sociología y la antropología médicas ${ }^{(32)}$. Pero es indispensable construir sociológicamente el campo de la salud y localizar ahí a la salud colectiva (es decir, construir el mapa de las relaciones de fuerza que sostiene con los otros integrantes del campo). No haber completado esta tarea explica la dificultad que se advierte en varios autores para ir más allá de las descripciones hasta ahora propuestas acerca de la situación de la salud colectiva, o del papel de las ciencias sociales dentro de ella.

En el desarrollo de este ejercicio de reflexividad, es indispensable recordar dos de las propiedades sociales de las ciencias sociales que, a los efectos de este trabajo, son fundamentales: a) sus productos se confunden con los del sentido común porque hablan de cuestiones de las que todo el mundo, en cierto sentido, es "experto". Gozan, por tanto, de muy poca autonomía, como ocurrió con la astronomía y la biología evolutiva en su momento; y b) la sociología médica conoce vertientes a veces más autónomas y a veces más heterónomas, en buena medida, en función del tipo de institución (universitaria o de gobierno) en la que se desarrolle. Las versiones más heterónomas de las ciencias sociales en salud producen especialistas más reconocidos o aceptados por el establishment médico o de salud pública, o más funcionales a este, lo que contribuye a reforzar la posición de desconocimiento de la sociología: "la verdad científica no se impone por sí misma, es decir, por la mera fuerza de la razón demostrativa (ni siquiera en los campos científicos). La sociología es socialmente débil y, tanto más, sin duda, cuanto más científica es"(11 p.154).

Dentro del campo médico o de la salud, las ciencias sociales juegan un papel subalterno, están Ilamadas a "colaborar"; y dentro del campo de las ciencias sociales, la salud ocupa uno de los últimos lugares en la jerarquía de los objetos legítimos de estudio. Habría que comprender que, dentro del campo médico, los científicos sociales gozan de poca autonomía pero son más tomados en cuenta; mientras que, dentro del campo académico, los antropólogos médicos y los sociólogos de la salud tienen más autonomía relativa pero poco impacto sobre el campo de la salud (esto es, sus descubrimientos y análisis no son traducidos fácilmente en políticas y programas de salud). Los más heterónomos -es decir, aquellos que cumplen básicamente la función de ejecutar la agenda de investigación del establishment biomédico- contribuyen a perpetuar una visión convencional de los problemas de salud y de la naturaleza y de las potencialidades (debidamente domesticadas) de las ciencias sociales.

La lógica de los campos ${ }^{(33),(34)}$ nos permite ubicar una de las principales tensiones a la que se ven sometidos los científicos sociales. Marsiglia ${ }^{(35)}$ reporta correctamente que, al ser llamados a "cooperar" con las ciencias de la salud, los científicos sociales suelen verse en la incómoda situación de tener que decir cosas "para resolver problemas": suele imponerse sobre ellos el mandato de ser "prácticos", de aportar soluciones y, por ende, de abandonar cualquier sofisticación teórica o metodológica que, bajo la lógica práctica prevaleciente en el campo de la salud, puede interpretarse como superflua. La subordinación de las ciencias sociales dentro del campo de la salud hace muy difícil tener en cuenta una propiedad más de aquellas, que Weber formuló con toda claridad en La ciencia como 
vocación, al establecer que ni las ciencias ni las ciencias sociales pueden decirnos qué debemos hacer. Su aporte estriba básicamente en métodos para pensar e investigar, en la disciplina para hacerlo, y la claridad para hacer mejores preguntas ${ }^{(36)}$. Y, al mismo tiempo, la falta de una teoría de los campos para objetivar la salud colectiva como parte del campo médico o de la salud (y como parte también del campo donde se disputa la definición del modelo de atención de la salud de las poblaciones -el campo de la salud poblacional- en el que también encontramos a la salud pública en sus diversas modalidades) explica algunas de las deficiencias que se encuentran en las respuestas y alternativas que formulan algunos autores cuando se trata de explicar y transformar la posición de subordinación de las ciencias sociales en su relación con las materias de salud. Por ejemplo, tras preguntarse si es posible producir un conocimiento social que no esté subordinado a las ciencias naturales, Marsiglia solo acierta a responder: "sí, si conseguimos mirar conjuntamente al objeto real..."(35 p.39).

Otra característica del campo médico o de la salud $-y$ de la salud colectiva dentro de él-, estrechamente relacionada con el punto anterior, se refiere a los cambios que se están observando en los posgrados de ciencias sociales y salud, y al papel que juegan en ello las agencias estatales que financian la investigación. Amélia $\operatorname{Cohn}^{(37)}$ señala otra tensión a la que están sujetos los científicos sociales en salud: la prisa que se les impone por completar las investigaciones que les encargan, debido a la urgencia por solucionar problemas, contraria al ritmo más pausado propio de las investigaciones sociológicas. Ello redunda en una pobre producción sociológica y en estudios "cuanti-cuali" dictados así por el establishment médico. Lo que se termina produciendo confirma la irrelevancia de las ciencias sociales y las "buenas razones" que tienen los biomédicos para no reconocerle un mayor estatus. La autora señala que la mayoría de los estudios en ciencias sociales en salud los hacen profesionales sin formación originaria en ciencias sociales. $\mathrm{Y}$, como en el caso anterior, la falta de una objetivación adecuada del campo de la salud explica la limitada solución que ofrece Cohn a la producción sistemática de la subordinación de las ciencias sociales en salud, y a las exigencias de rapidez y eficiencia que se les imponen: "la busca de interlocución entre estrategias distintas de apropiación científica de la realidad, solo puede ocurrir si ellas fueran respetadas en sus especificidades y exigencias"(37 p.17). La cuestión aquí, al igual que en el caso del ejemplo del párrafo anterior, nos parece que no solo es Ilamar al respeto de las especificidades de las diversas ciencias de la salud, o al trato igualitario entre las diversas disciplinas, sino sobre todo explicar por qué dicho respeto o trato igualitario no ocurre. Necesitamos preguntarnos, en vías a una objetivación completa, por qué se produce y reproduce aquella forma fallida de ciencias sociales y sus efectos reproductores de la subordinación.

En el esfuerzo de construcción del campo médico o de la salud, un paso necesario sería objetivar sociológicamente la propia salud colectiva -en tanto uno de los enfoques que forman parte del campo- para poder desencantar dicha perspectiva e identificar la naturaleza social de su fuerza pero también de sus debilidades. Es notable, por ejemplo, la abundancia de artículos publicados, sobre todo en Brasil (donde el enfoque es más vigoroso), que reivindican el carácter crítico y emancipador de la salud colectiva y que identifican diversos "adversarios" contra los cuales se lucha: la epidemiología convencional, los métodos cuantitativos, la ideología y práctica del productivismo científico, la salud pública dominante y acrítica, etc. Todas estas batallas se formulan desde el lugar de la subalternidad, desde la necesidad de luchar contra los modelos que dictan las reglas que estructuran el campo de la salud y, dentro de él, el campo de la salud poblacional. Y desde ahí se han hecho contribuciones importantes que apuntan justo hacia una sociología de la salud colectiva al ilustrar diversas transformaciones de orden material (por ejemplo, el abaratamiento de los posgrados o la imposición de esquemas de producción académica y evaluación) que se asocian a las luchas internas 
dentro de la salud colectiva y dentro del campo de la salud poblacional(38),(39).

Pero una buena parte de las dificultades enfrentadas hasta ahora estriba en las imprecisiones, motivadas por la pasión y el compromiso de diversos autores respecto a sus propias convicciones, con que se ha propuesto utilizar la noción de "campo". Desde la salud colectiva abundan las declaraciones que la reivindican como un "campo científico" ${ }^{\prime(40)}$. Se trata de una caracterización equivocada por cuanto se encuentra ausente la dimensión de conflicto propia de todo campo: ¿qué está en juego, cuáles son los capitales en disputa dentro de la salud $\mathrm{CO}^{-}$ lectiva? Los autores no solo ignoran el carácter agonista que deben identificar en un campo, sino que al mismo tiempo ofrecen una descripción "hagiográfica" del mismo: hablan de "la constitución de la salud colectiva, teniendo en cuenta sus fecundos diálogos con la salud pública y con la medicina social..." ${ }^{\prime 40}$ p.309). En realidad, la relación entre esas tres perspectivas ha sido mucho más de conflicto que de diálogo profundo. La acepción de la salud colectiva como un "campo" quizá podría aceptarse siempre que se piense como sinónimo de "perspectiva disciplinaria". Pero no como lo entiende el estructuralismo genético de Bourdieu. Desde este último enfoque, la salud colectiva estaría formada por un conjunto de actores, individuales e institucionales, que forman parte de un campo mayor -el de la salud poblacional- que, a su vez, es parte de uno más amplio: el campo médico o de salud en general. En el campo de la salud poblacional también hay otro conjunto de actores -los representantes del enfoque de la salud pública, la Organización Mundial de la Salud, la Organización Panamericana de la Salud, diversas universidades, etc.- y lo que se disputa es la capacidad de dictar e imponer las normas mediante las cuales han de concebirse los problemas de salud de las poblaciones y las políticas que le son pertinentes, la manera de estudiarlos y la manera de evaluar la calidad científica de tales investigaciones (reglas de sobrevivencia académica) $y$, desde luego, la manera misma en que ha de llamarse el campo ${ }^{(41)}$. Sería un campo en el que, claramente, la posición dominante (aunque no necesariamente hegemónica) está ocupada por la salud pública tradicional y su visión de las cosas, y contra la cual se articulan las críticas y las contrapropuestas que se formulan desde la salud colectiva.

Tal caracterización está mejor lograda en el trabajo de Bertol Leal y Camargo Junior ${ }^{(42)}$, que cuestiona la pertinencia de caracterizar la salud colectiva como un campo científico (dado que la práctica está implicada en la salud colectiva de una manera diferente a como lo está en el campo científico) y sugiere la necesidad de acceder a un concepto de campo más amplio ${ }^{[e]}$. El campo de salud, señalan, está formado:

...por las diferentes instituciones productoras de conocimiento (asociaciones, universidades, centros formadores y de investigación), los diferentes profesionales de la salud (médicos, enfermeros, entre otros), gobernantes, administradores y tecnoburócratas, los productores de insumos y tecnologías, los usuarios (pacientes, enfermos) y varias instituciones, tales como hospitales, puestos de salud o unidades básicas, secretarías estatales y municipales de salud, asociaciones de corporaciones profesionales (como sindicatos, consejos profesionales y de especialidades), asociaciones de instituciones prestadoras de servicios (como de hospitales y de laboratorios), asociaciones y consejos de representación gubernamental (como el consejo de secretarios municipales y estatales de salud), asociaciones de usuarios (tales como ONG de sida, asociaciones diversas de enfermos que tienen las mismas patologías), además de consejos institucionalizados de participación de diferentes actores (consejos municipales y estatales de salud, consejos gestores de servicios). ${ }^{(42)}$

Los autores Ilaman a no confundir la salud colectiva en Brasil con la Reforma Sanitaria en ese país. De igual manera, en 
el esfuerzo de objetivación de la salud colectiva, como parte del campo de la salud en América Latina, es necesario no confundirla con el caso concreto de Brasil, aun cuando, sin duda, es su principal referente. Si bien, nos parece, hay cierta ambigüedad en la localización de la salud colectiva (a veces se la cita como parte del campo de la salud, y a veces se habla del campo de la salud colectiva) los autores detectan un elemento central del campo que, sin embargo, no logran objetivar del todo: la illusio, es decir, la creencia (incluso apasionada), de que lo que está en juego es importante y de que vale la pena jugarse por ello. Señalan que una de las características del campo es "su carácter militante en la disputa por la validación de las Verdades que más responden a las necesidades sociales; se trata de un movimiento de tensión que genera energías de renovación" ${ }^{\prime(42 \text { p.62). }}$. Pero no logran distanciarse de lo que les apasiona: en lugar de objetivar dicho carácter militante, acreditan estar plenamente tomados por el campo al reivindicar como un valor su carácter agonista. Pensamos que la objetivación del campo debe ofrecer también al escrutinio sociológico aquello que a los actores más comprometidos podría parecerles sus valores más preciados: su convicción, su militancia. El obstáculo mayor para objetivar un campo al que se pertenece es el hecho de estar tomado por el campo:

...el punto de vista que objetiva los puntos de vista y los constituye como tales [...] entraña la sustitución de la visión polémica, parcial y arbitraria de los agentes mismos [...] por una visión comprensiva e indulgente de acuerdo con la fórmula 'comprender es perdonar' de las diferentes posiciones y tomas de posición [lo] que no implica de ningún modo la supresión de las diferencias de puntos de vista. Además, lejos de conducir, como podría creerse... a un relativismo que no da la razón a ninguno de los competidores por la verdad, la construcción del campo permite establecer la verdad de las diferentes posiciones. ${ }^{(44}$ p.99)
Otros trabajos han documentado no solo el carácter subalterno de la salud colectiva, sino además una suerte de crisis de identidad "manifiesta en su fragmentación y dilución como campo científico" ${ }^{\prime(45)}$. Se señala que, a pesar de sus desarrollos teóricos y del uso sofisticado de conceptos sociológicos, la salud colectiva sigue siendo subalterna y en varios sentidos instrumental a una concepción biologicista dominante, pragmática, positivista y "del gusto de los anglosajones". De tal manera que, junto a los trabajos reivindicativos, también están aquellos que advierten señales de desencanto, en el sentido de que no se cumplió la promesa que la salud colectiva albergaba, y de que no hay muchas señales de que las cosas vayan a cambiar.

Para objetivar la salud colectiva, entonces, habría que construir el campo de luchas dentro del cual sería posible entender sus movimientos, sus reivindicaciones... y sus silencios. Lo que también está en juego en el campo de la salud es el monopolio del enfoque legítimo con el que han de estudiarse los determinantes de la salud y el lugar y papel de las ciencias sociales en ello. El campo médico o de la salud es, a su vez, un subcampo del campo del poder, y tiene claras vinculaciones con el campo académico y el campo editorial ${ }^{(46)}$. Contemplar esto último nos permitiría avanzar en la objetivación de la salud colectiva, identificando su lugar en las diferentes jerarquías de estructuras de poder en las que está inmersa, y diferenciando los múltiples actores en su interior. En este sentido, en términos profesionales, la fracción dominante de este subcampo dominado parece estar formada por especialistas en salud con cierta formación en ciencias sociales, mientras que los especialistas en ciencias sociales con interés en la salud ocupan una posición secundaria ${ }^{(37),(47)}$; $y$, en términos regionales, la fracción dominante de este mismo subcampo se ubicaría sobre todo en Brasil, mientras que los demás países de la región ocuparían una posición satelital ${ }^{(10),(48)}$.

Una vez ubicada la salud colectiva dentro del campo médico o de la salud, y habiendo identificado las principales lógicas 
de acción que estructuran las luchas en su interior, debería ser posible avanzar hacia una sociología de la salud colectiva que incluya un análisis de las condiciones de posibilidad de transformación de los principales objetos en disputa: por ejemplo, el productivismo académico, el desinterés activo del establishment por el estudio de lo social en sus vertientes más críticas (y el fomento de unas ciencias sociales sin mayor capacidad de cuestionamiento), o la limitada incidencia en políticas públicas, entre otros. La objetivación de estos aspectos, a su vez, nos adentraría en terrenos incómodos pero cuya exploración es imprescindible.

Por ejemplo, desde la salud colectiva se ha objetado el productivismo académico al que se hallan sometidos sus representantes, producto de la imposición de un estilo de evaluación académica que se dicta desde el enfoque biomédico y sanitarista dominante y que es apoyado por el Estado en beneficio de la industria editorial. Se habla incluso -vieja lucha entre los que nos dedicamos a la sociología médica- de la necesidad de reivindicar el libro frente a los artículos, pues aquel es una herramienta más apropiada para expresar los hallazgos de las ciencias sociales. Sin embargo, las críticas al sistema establecido (por demás pertinentes) permanecen en silencio respecto a un aspecto crucial: mediante qué indicadores y a través de qué sistema de evaluación institucional podríamos diferenciar la investigación de calidad, y sus productos, de la investigación mediocre o francamente mala $^{(49)}$. Es correcta la crítica de que el sistema productivista confunde calidad con cantidad: a falta de capacidad para evaluar la primera opta por concentrarse en la segunda (o bien privilegia el productivismo por diversos intereses) $)^{(38),(46)}$. Lo que hay que preguntarse ahora es por qué la crítica a ese modelo no ha formulado una alternativa viable y de qué manera esa reiterada postura "crítica-sin-alternativas" contribuye a reproducir su situación de subalternidad al mantenerse en una posición de "desprestigio" dentro del campo ${ }^{(49)}$. En la descripción de lo que es una investigación de "calidad" se juega, desde luego, la primacía del grupo sanitarista tradicional sobre el grupo de especialistas en ciencias sociales. Mientras el primero propende a demandar que las investigaciones "sirvan" para solucionar problemas concretos, el segundo grupo, en cambio, sin necesidad de aportar para las soluciones de problemas concretos, propende a reivindicar el carácter sobre todo clarificador de sus investigaciones. Pero, además, se juegan otras cosas importantes, como la justificación para que la investigación social en salud reciba financiamiento público (si es de calidad) o no (si no lo es). Probablemente, una adecuada objetivación de la salud colectiva nos aportará nuevos elementos de sociología del conocimiento que nos permitan descubrir qué nos impide subvertir este orden y proponer alternativas, salvando estos escollos.

Por otra parte, una sociología de la salud colectiva debería llevarnos a cuestionar el origen social del Ilamado "movimiento" por la investigación cualitativa y su obstinada crítica de la investigación cuantitativa, como si esta última fuera siempre mala ciencia, o como si lo cualitativo garantizara de por sí una ciencia de calidad(50),(51),(52). La investigación cualitativa que se publica en la región con frecuencia está desprovista de sólidos anclajes teóricos y a veces carece de conceptos básicos que la alejen de la sociología espontánea y de las interpretaciones de mero sentido común ${ }^{(53)}$. Una subversión de las relaciones de poder dentro del campo de la salud debería pasar por la demostración empírica, basada en evidencias pertinentes, de que la ciencia que se propugna es superior a la dominante. Pero lejos de privilegiar el debate y la refutación, lo que abunda son declaraciones de principios y proclamas autocelebratorias que en realidad mueven poco el campo. ¿Qué impulsa a los defensores de este "movimiento"? ¿ isu "lucha", en realidad, no perpetúa su condición de subalternidad? A los efectos de este trabajo, la cuestión es fundamental pues lo que está en juego, no lo olvidemos, es la posibilidad de desarrollar una ciencia social aplicada a la salud que resulte verdaderamente esclarecedora y que, por tanto, pueda des-cubrir los determinantes del conocimiento que se produce, 
y del que se omite. El ejemplo que hemos puesto lo ilustra con claridad: avanzar sustantivamente en el estudio sociológico de los determinantes de la salud-enfermedad exige poner bajo examen los Ilamados factores objetivos (primera aproximación), el carácter socialmente construido de las categorías de conocimiento (segunda aproximación), y las luchas y relaciones de poder que determinan la viabilidad o no de tales categorías (tercera aproximación).

\section{CONCLUSIÓN}

La salud colectiva está en buena posición para intentar la objetivación de sí misma. Sabe del potencial crítico de las ciencias sociales, y sabe de las consecuencias que resultan si se las aplica sin un horizonte emancipador. Pero para hacer sociología de la salud colectiva desde la salud colectiva hay que enfrentar un formidable obstáculo epistemológico: el habitus de sus integrantes que los predispone a criticar a los poderes establecidos y, con ello, la propensión a dejar de observarse y criticarse a sí mismos. La suma de reflexiones importantes que se han hecho en torno a la salud colectiva, que apuntan a su cooptación o al debilitamiento de la perspectiva, solo podrá completarse en la medida en que se acceda a la plena objetivación reflexiva del enfoque. La teoría de los campos, de Bourdieu, puede ser una herramienta muy útil en este esclarecimiento. Pero, sobre todo, lo será la voluntad de someter a prueba empírica las hipótesis y los "hallazgos" que se vayan presentando. En este artículo no hemos hecho sino identificar algunos aspectos que dicho esfuerzo de objetivación debe tener presentes. Pero el proyecto, como tal, sigue pendiente de realización.

\section{NOTAS FINALES}

a. En otro trabajo mostré esto mismo con relación al maltrato que sufren las mujeres en los servicios de salud durante la atención del parto: mientras el objeto sea definido bajo la hegemonía del campo médico, el asunto se mantiene como un problema de "calidad de la atención" o como un problema de "deshumanización" de la medicina, término que demuestra la orfandad de ciencias sociales para su conceptualización. En cambio, si para estudiar dicho problema se objetiva al propio campo médico en términos sociológicos, entonces se pueden dilucidar los factores más profundos que lo estructuran $^{(12),(13)}$.

b. Una materia de estudio fascinante es la enorme popularidad que están adquiriendo estas "nuevas medicinas" así como las disputas epistémicas y prácticas que existen entre ellas y la medicina alopática ${ }^{(28)}$. En la construcción de este objeto es necesario sustraerse de las mutuas acusaciones que se lanzan desde ambos agregados y advertir que no toda la medicina alternativa es "pseudociencia", al tiempo que también dentro de la medicina alopática se observan creencias y prácticas no científicas.

c. Un grupo de investigadores y docentes de la UNAM ha creado una página en Facebook llamada "No a la pseudociencia en la UNAM" (NoPseudociencia) para trabajar activamente en contra de las nuevas formas de medicalización, entre otros objetivos. El Center for Inquiry, de EE.UU., también libra una batalla contra las "medicinas alternativas complementarias". En la construcción del campo de la salud, habría que incluir a estos nuevos actores. d. Para una revisión de las controversias epistemológicas que suscita el enfoque del constructivismo social vale la pena revisar el debate que al respecto sostuvieron Bury, Nicolson y McLaughlin 29$),(30),(31)$.

e. La definición coincide bastante bien con la que ofrecí en otro trabajo acerca del campo médico en México: “El campo médico está formado por el conjunto de organizaciones, instituciones y actores de la salud que, ubicados en diferentes posiciones, mantienen entre sí relaciones de fuerza orientadas a conservar, adquirir o transformar esa forma de capital específico que consiste en la capacidad de imponer los esquemas dominantes de definición, percepción y apreciación de las materias propias de la agenda sanitaria, así como de la acción (política, comercial, científica, profesional) que deriva de ello. Así entendido, junto con las instituciones de salud propiamente tales, forman parte del campo médico la industria farmacéutica y la de aparatos médicos, las aseguradoras, y las formas de medicinas subalternas, tales como la homeopatía, la medicina quiropráctica, etc. Y, de manera muy relevante para esta investigación, son parte del campo médico las instituciones que forman a los nuevos cuadros profesionales que eventualmente pasarán a formar parte del campo, los agentes que operan estas instituciones, así como los profesores y estudiantes de todas estas especialidades" ${ }^{\prime \prime}(3$ p.342).

\section{REFERENCIAS BIBLIOGRÁFICAS}

1. Straus R. The nature and status of medical sociology. American Sociological Review. 1957;22(2):200-204. 
2. Nunes ED. Saúde Coletiva: História de uma idéia e de um conceito. Saúde e Sociedade. 1994;3(2):5-21.

3. De la Garza-Toledo E, Leyva G. Introducción. En: De la Garza-Toledo E, Leyva G, (eds). Tratado de metodología de las ciencias sociales: perspectivas actuales. México: Fondo de Cultura Económica, Universidad Autónoma Metropolitana; 2012. p. 19-32.

4. Minayo MCS. Análise qualitativa: teoría, passos e fidedignidade. Ciencia \& Saúde Colectiva. 2012;17(3):621-626.

5. Popper KR. La lógica de las ciencias sociales. En: Popper KR, Adorno TW, Dahrendorf R, Habermas J. La lógica de las ciencias sociales. México: Grijalbo; 1978. p. 9-27.

6. Gomes MHA, Silveira C. Sobre o uso de métodos qualitativos em Saúde Coletiva, ou a falta que faz uma teoria. Revista de Saúde Pública. 2012;46(1):160-165.

7. World Health Organization. New public health and WHO's Ninth General Programme of Work: a discussion paper. Geneva: WHO; 1995.

8. Harvey G, Marshall RJ, Jordan Z, Kitson AL. Exploring the hidden barriers in knowledge translation: A case study within an academic community. Qualitative Health Research. 2015;25(11):1506-1517.

9. Currie G, El Enany N, Lockett A. Intra-professional dynamics in translational health research: The perspectiveof social scientists. Social Science \& Medicine. 2014; 114:81-88

10. Luz MT. Especificidade da contribuição dos saberes e práticas das Ciências Sociais e Humanas para a saúde. Saúde e Sociedade. 2011;20(1):22-31.

11. Bourdieu P. El oficio de científico: Ciencia de la ciencia y reflexividad. Barcelona: Anagrama; 2003.

12. Castro R. Génesis y práctica del habitus médico autoritario en México. Revista Mexicana de Sociología. 2014;76(2):167-197.

13. Castro R, Erviti J. Sociología de la práctica médica autoritaria: Violencia obstétrica, anticoncepción inducida y derechos reproductivos. Cuernavaca: CRIM-UNAM; 2015.

14. Castro R. Teoría social y salud. Buenos Aires: Lugar Editorial, CRIM-UNAM; 2011.

15. Organización Mundial de la Salud. Subsanar las desigualdades en una generación: Alcanzar la equidad sanitaria actuando sobre los determinantes sociales de la salud [Internet]. Ginebra: OMS; 2008 [citado 10 oct 2015]. Disponible en: http://goo.gl/W1ZsKz.

16. Asociación Latinoamericana de Medicina Social. Taller Latinoamericano sobre Determinantes Sociales de la Salud [Internet]. 2008 [citado 10 oct 2015]. Disponible en: https://goo.gl/G9cW08.

17. Birn AE. ¿Politizándolo o puliéndolo? Subsanar las desigualdades en una generación: alcanzar la equidad sanitaria actuando sobre los determinantes sociales de la salud. Medicina Social. 2009;4(3):189-201.
18. Navarro $\mathrm{V}$. What we mean by social determinants of health. International Journal of Health Services. 2009;39(3):423-441.

19. Garbois JA, Sodré F, Dalbello-Araujo M. Social determinants of health: the "social" in question. Saúde e Sociedad. 2014;23(4):11-19.

20. Lemert EM. Social pathology: a systematic approach to the theory of sociopathic behavior. Los Angeles: McGraw-Hill; 1951.

21. Lemert EM. Beyond Mead, the societal reactions to deviance. Social Problems. 1974;21:457-468.

22. Becker HS. Outsiders: Studies in the sociology of deviance. New York: The Free Press; 1963.

23. Scheff TJ. El rol del enfermo mental. Buenos Aires: Amorrortu; 1973.

24. Good BJ. Medicia, racionalidad y experiencia: Una perspectiva antropológica. Barcelona: Edicions Bellaterra; 2003.

25. Freidson E. La profesión médica: Un estudio de sociología del conocimiento aplicado. Barcelona: Ediciones Península; 1978.

26. Erviti J, Castro R, Sosa I. Las luchas clasificatorias en torno al aborto, el caso de los médicos en hospitales públicos de México. Estudios Sociológicos. 2006;24(72): 637-665.

27. Moynihan R, Heath I, David H. Selling sickness, the pharmaceutical industry and disease mongering. British Medical Journal. 2002;324:886-891.

28. Brosnan C. 'Quackery' In the Academy? Professional knowledge, autonomy and the debate over complementary medicine degrees. Sociology. 2015;49(6): 1047-1064.

29. Bury MR. Social constructionism and the development of medical sociology. Sociology of Health and Illness. 1986;8(2):137-169.

30. Nicolson M, McLaughlin C. Social constructionism and medical sociology: a reply to M.R. Bury. Sociology of Health and Illness. 1987;9(2):107-126.

31. Bury MR. Social constructionism and medical sociology: A rejoinder to Nicolson and McLaughlin. Sociology of Health and Illness. 1987;9(4):439-441.

32. Loyola MAR. A saga das ciências sociais na área da Saúde Coletiva: elementos para reflexão. Physis, Revista de Saúde Coletiva. 2008;18(2):251-275.

33. Bourdieu P. Homo academicus. Buenos Aires: Siglo XXI Editores; 2008.

34. Bourdieu P. Una invitación a la sociología reflexiva. Buenos Aires: Siglo XXI Editores; 2005

35. Marsiglia RM. Temas emergentes em Ciências Sociais e Saúde Pública/Coletiva: a produção do conhecimento na sua interface. Saúde e Sociedade. 2013;22(1):32-43. 
36. Weber M. El político y el científico. Madrid: Alianza Editorial; 1979.

37. Cohn A. Ciências Sociais e Saúde Pública/Coletiva: a produção do conhecimento na sua interface. Saúde e Sociedade. 2013;22(1):15-20.

38. Luz MT. Prometeu acorrentado: Análise da categoría productividade e as condições atuais da vida académica. Physis, Revista de Saúde Coletiva. 2005;15(1):39-57.

39. Loyola MA. O lugar das Ciencias Sociais na Saúde Coletiva. Saúde e Sociedade. 2012;21(1):9-14.

40. Paim JS, Almeida-Filho N. Saúde coletiva: uma "nova saúde pública" ou campo aberto a novos paradigmas? Revista de Saúde Pública. 1998;32(4):289-316.

41. Minayo MCS. A produção de conhecimentos na interface entre as ciências sociais e humanas e a Saúde Coletiva. Saúde e Sociedade. 2013;22(1):21-31.

42. Leal MB, Camargo Junior KR. Saúde coletiva em debate: reflexões acerca de um campo em construção. Interface-Comunicação, Saúde, Educação. 2012;16(40): 53-65.

43. Castro R. Pautas de género en el desarrollo del habitus médico: los años de formación en la escuela de medicina y la residencia médica. Salud Colectiva. 2014;10(3):339-351

44. Bourdieu P. Los usos sociales de la ciencia. Buenos Aires: Ediciones Nueva Visión; 2000.
45. Campos GWS. Saúde pública e saúde coletiva: campo e núcleo de saberes e práticas. Ciencia \& Saúde Coletiva. 2000;5(2):219-230.

46. Martinovich V, Arakaki J y Spinelli H. Diez años de Salud Colectiva: una aproximación a las reglas del juego del campo editorial científico. Salud Colectiva. $2014 ; 10(1): 5-13$.

47. Canesqui AM. Sobre a presença das Ciências Sociais e Humanas na Saúde Pública. Saúde e Sociedade. 2011;20(1):16-21.

48. Canesqui AM. Produção científica das Ciências Sociais e Humanas em saúde e alguns significados. Saúde e Sociedade. 2012;21(1):15-23.

49. Bosi MLM. Pesquisa qualitativa em saúde coletiva: panorama e desafios. Ciencia \& Saúde Coletiva. 2012;17(3):575-586.

50. Almeida-Filho N. Epidemiología sin números. Washington DC: OPS; 1992.

51. Almeida-Filho N. Por una epidemiología con (más que) números: cómo superar la falsa oposición cuantitativo-cualitativo. Salud Colectiva. 2007;3(3):229-233.

52. Diez-Roux AV. En defensa de una epidemiología con números. Salud Colectiva. 2007;3(2):117-119.

53. Minayo MCS. Los conceptos estructurantes de la investigación cualitativa. Salud Colectiva. 2010;6(3): 251-261.

\section{FORMA DE CITAR}

Castro R. De la sociología en la medicina a la sociología de la salud colectiva: apuntes para un necesario ejercicio de reflexividad. Salud Colectiva. 2016;12(1):71-83.

Recibido: 10 de diciembre de 2015 | Aprobado: 6 de febrero de 2016

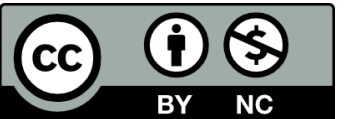

Este obra está bajo una licencia de Creative Commons Reconocimiento-NoComercial 4.0 Internacional. Reconocimiento - Permite copiar, distribuir y comunicar públicamente la obra. A cambio, se debe reconocer y citar al autor original. No Comercial - Esta obra no puede ser utilizada con finalidades comerciales, a menos que se obtenga el permiso.

http://dx.doi.org/10.18294/sc.2016.859 